\title{
Rotulagem nutricional: você sabe o que está comendo?
}

Nutritional labelling: do you know that are you eating?

\section{Autores | Authors}

\section{Giovanna da Silva CAVADA}

Universidade Federal de Pelotas (UFPel) Departamento de Nutrição Pelotas/RS - Brasil e-mail: gsc.nutri@hotmail.com

Flávia Fernandes PAIVA

Universidade Federal de Pelotas (UFPel) Departamento de Ciência e Tecnologia Agroindustrial

Pelotas/RS - Brasil

e-mail: fafernandespaiva@yahoo.com.br

\section{Elizabete HELBIG}

Universidade Federal de Pelotas (UFPel) Departamento de Nutrição Pelotas/RS - Brasil e-mail: helbignt@gmail.com

凶 Lúcia Rota BORGES

Universidade Federal de Pelotas (UFPel) Departamento de Nutrição Campus Anglo

Rua Gomes Carneiro, 01 CEP: 96010-610

Pelotas/RS - Brasil e-mail: luciarotaborges@yahoo.com.br

Autor Correspondente / Corresponding Author Publicado / Published: dezembro/2012

\section{Resumo}

O rótulo tem por função orientar o consumidor sobre os constituintes dos alimentos, promovendo escolhas alimentares saudáveis. No entanto, isto não significa que os consumidores o estejam utilizando como uma ferramenta para a escolha dos alimentos que deverão compor sua dieta e, assim, reduzir os excessos alimentares e os danos ocasionados à saúde. Este trabalho, um estudo transversal, avaliou o hábito de leitura dos rótulos de consumidores em uma rede de supermercados de Pelotas-RS. Verificou-se que $48,13 \%$ dos entrevistados avaliam os rótulos dos alimentos. Os usuários da rotulagem são, na sua maioria, mulheres, jovens e com curso superior. Houve associação significativa entre hábito de leitura e influência na compra dos produtos, o que mostra a importância da rotulagem como um instrumento no ato da compra, uma vez que representa um elo de comunicação entre o consumidor e o produto; além disso, se o rótulo é bem compreendido, permite escolhas alimentares mais criteriosas.

Palavras-chave: Rotulagem de alimentos; Informação nutricional; Comportamento do consumidor.

\section{Summary}

The label has the task of guiding the consumer on the constituents of food, promoting healthy food choices. However, this does not mean that consumers are using as a tool to choose which foods should make up your diet, and thus reduce overeating and damages health. This is a cross-sectional study, which evaluated the habit of reading labels for consumers in a supermarket chain in Pelotas, RS. It was found that $48.13 \%$ of respondents evaluate food labels. Users of labeling are mostly women, young people and university graduates. A significant association between reading habits and influence on the purchase of products, which shows the importance of labeling as a tool in the purchase, since it represents a communication link between the consumer and the product is well understood and allows choices food more insightful.

Key words: Nutritional labeling; Nutritional information; Consumer's behavior. 


\section{Introdução}

As modificações no padrão alimentar da população - evidenciadas pelo consumo de dietas com alta densidade energética, ricas em gordura de origem animal e com baixo teor de fibras - associadas à maior prevalência do sedentarismo, ao tabagismo e ao abuso excessivo de álcool têm levado ao aumento da incidência de algumas doenças, entre as quais as doenças cardiovasculares, a obesidade e o diabetes mellitus (GIMENO et al., 2011; LENZ et al., 2009). Segundo Souza et al. (2011), o consumo alimentar é um determinante da saúde, cujo caráter positivo ou negativo depende de informações adequadas, sendo de fundamental importância intervenções de educação nutricional que auxiliem a população na escolha de alimentos mais saudáveis.

A rotulagem nutricional é definida como toda a descrição destinada a informar o consumidor sobre as propriedades nutricionais de um alimento, compreendendo a declaração de valor energético e os principais nutrientes. No entanto, é necessário que estas informações sejam compreendidas por todos aqueles que as utilizam (ANVISA e UnB, 2005; CÂMARA et al., 2008; SOUZA et al., 2011). As informações fornecidas por meio da rotulagem contemplam um direito assegurado pelo Código de Defesa do Consumidor, o qual determina que a informação sobre produtos deve ser clara e com especificação correta de quantidade, composição e qualidade, bem como sobre os riscos que possam apresentar (BRASIL, 1990; CÂMARA et al., 2008).

No Brasil, a rotulagem nutricional é regulamentada pelas Resoluções de Diretoria Colegiada (RDCs) 360/03 e 359/03 da Agência Nacional de Vigilância Sanitária (ANVISA). Nesse sentido, devem ser declaradas, segundo a RDC 360/03, as quantidades por porção e a porcentagem do valor diário dos seguintes componentes: valor energético, teor de carboidratos, proteínas, gorduras totais, gorduras saturadas, gorduras trans, fibras alimentares e sódio (BRASIL, 2003b; LOBANCO et al., 2009). A RDC 359/03 estabelece as medidas e porções, incluindo a medida caseira e sua relação com a porção correspondente em gramas ou mililitros, detalhando também os utensílios utilizados com suas capacidades aproximadas. As porções indicadas nos rótulos de alimentos e bebidas foram determinadas com base em uma dieta de $2.000 \mathrm{kcal}$, considerando uma alimentação saudável (BRASIL, 2003a; LOBANCO et al., 2009).

Os rótulos são elementos de comunicação entre o produto e os consumidores, e devem auxiliá-los na decisão de compra, aumentando a eficiência do mercado e o bem-estar do consumidor (MACHADO et al., 2006). No entanto, o fato de a rotulagem ser obrigatória não significa que os consumidores a estejam utilizando como uma ferramenta para a escolha dos alimentos que devem compor sua dieta habitual e, assim, reduzir os excessos alimentares e, consequentemente, os danos ocasionados à saúde (MACHADO et al., 2006).

Em função da possibilidade de a alimentação inadequada trazer inúmeros riscos à saúde, é de extrema importância avaliar o conhecimento da população frente à rotulagem. Segundo dados do Ministério da Saúde, metade das pessoas que costumam ler os rótulos dos alimentos que consomem não compreendem adequadamente o significado destas informações (ANVISA e UnB, 2005).

Diante do exposto, o objetivo deste estudo foi verificar o hábito de leitura e o conhecimento das informações nutricionais contidas nos rótulos de alimentos por consumidores de uma rede de supermercados da cidade de Pelotas-RS.

\section{Material e métodos}

Foi realizado um estudo transversal durante os meses de abril a junho de 2011, com consumidores de uma rede de supermercados com filiais distribuídas na cidade de Pelotas-RS. Foram incluídos neste estudo clientes com idade igual ou superior a 18 anos, que foram entrevistados. Os dados foram coletados por entrevistadores treinados e as entrevistas foram realizadas nos dias úteis da semana, nos turnos manhã e tarde.

Aplicou-se um questionário com adaptações ao proposto por Cassemiro et al. (2006). Os participantes foram submetidos a perguntas referentes aos seguintes tópicos: variáveis socioeconômicas (gênero, idade, escolaridade e estado civil); hábito de leitura dos rótulos; compreensão das informações, e influência das informações na escolha dos produtos. O estado civil foi classificado em 'com companheiro' e 'sem companheiro', sendo que os viúvos e separados/divorciados foram agrupados junto aos 'sem companheiro'.

Para garantir a aleatoriedade do estudo, foram abordados os clientes que estavam na fila do caixa, aguardando o momento para pagar, sendo entrevistado o último cliente da fila, similarmente à metodologia descrita por Silva (2003). O cálculo do tamanho da amostra foi feito levando-se em consideração a população da cidade de Pelotas, sendo estimado um total de 246 clientes, considerando um erro máximo de $5 \%$ e nível de confiança de $95 \%$. Este total foi então distribuído proporcionalmente entre as filiais que compõem essa rede de supermercados.

Os dados foram tabulados no programa Excel 2007. As análises estatísticas foram realizadas no programa STATA versão 10.0. A análise descritiva das variáveis foi apresentada por meio de médias com seus respectivos desvios padrões. O teste de Qui-quadrado $\left(\chi^{2}\right)$ foi utilizado para testar a associação entre as 
variáveis. O nível de significância utilizado para todos os testes foi de $5 \%$.

O Comitê de Ética em Pesquisa da Faculdade de Medicina da Universidade Federal de Pelotas aprovou a realização desta pesquisa (OF.36/11). O Termo de Consentimento Livre e Esclarecido foi entregue ao cliente no início do estudo.

\section{Resultados e discussão}

Participaram do estudo 241 consumidores, com idade média de 42,46 anos $( \pm 14,88)$, sendo a maioria do gênero feminino (77,59\%). Quanto à escolaridade, 40,25\% dos entrevistados possuíam Ensino Superior. A Tabela 1 apresenta as características da população estudada.

Em relação ao hábito de leitura dos rótulos, 48,13\% ( $n=116)$ dos entrevistados afirmaram ler as informações presentes, 27,80\% ( $n=67)$ não liam e 24,07\% ( $n=58)$ liam às vezes. Dentre aqueles que tinham o hábito da leitura ou que liam às vezes, $91,96 \%(n=160)$ consideravam importantes estas informações.

Neste trabalho, pôde-se observar que a maioria dos entrevistados afirmou consultarem os rótulos, o que está de acordo com os achados de Felipe et al. (2003) e Cassemiro et al. (2006), evidenciando uma maior preocupação por parte da população de conhecer a qualidade nutricional dos alimentos.

Avaliando-se o comportamento de 300 consumidores frente à rotulagem na cidade de Feira de Santana-BA, Machado et al. (2006) encontraram uma prevalência de leitura em torno de $80,0 \%$, sendo que, destes, 52,0\% consultavam os rótulos constantemente. Pinheiro et al. (2011) encontraram uma prevalência de leitura de 85,40\%. Em estudo de Monteiro et al. (2005), com 187 indivíduos, $74,80 \%$ consultavam os rótulos durante as compras.

Ao se fazer a associação entre características socioeconômicas e hábito de leitura dos rótulos, os resultados apontaram diferença estatística apenas na variável 'escolaridade' (Tabela 2), evidenciando que indivíduos com nível de escolaridade mais alto possuem maior hábito desta leitura.

Estes dados são semelhantes aos encontrados por Cassemiro et al. (2006), que observaram uma maior utilização da informação nutricional conforme aumenta o grau de instrução, demonstrando que 79,0\% dos usuários que apresentavam Curso Superior possuíam maior interesse pela leitura dos rótulos. Monteiro et al. (2005) também encontraram uma relação direta e significativa entre hábito de leitura dos rótulos e escolaridade.

Quanto à compreensão do conteúdo dos rótulos, foi feita a associação entre hábito de leitura e compreensão das informações. Os resultados apontam que, dentre os entrevistados que costumavam ler os rótulos, 61,21\% ( $n=71)$ compreendiam as informações. Ao se avaliar a relação entre escolaridade e compreensão das informações, os achados evidenciam que mesmo sendo predominante o Ensino Superior para os indivíduos que compreendem estas informações (48,08\%), os dados não foram significativos $(p=0,459)$. Diversamente, no estudo de Pinheiro et al. (2011), houve relação entre grau de escolaridade e compreensão das informações.

É importante ressaltar que o rótulo nutricional é um instrumento fundamental no momento da compra dos alimentos, uma vez que representa um elo de comunicação entre o consumidor e o produto, e, se o rótulo é bem compreendido, permite escolhas alimentares
Tabela 1. Características da amostra $(n=241)$. Pelotas-RS, 2011.

\begin{tabular}{lcc}
\multicolumn{1}{c}{ Características } & $\mathbf{n}^{*}$ & $\%$ \\
Gênero & & \\
Masculino & 54 & 22,41 \\
Feminino & 187 & 77,59 \\
Faixa etária & & \\
18 a 40 anos & 112 & 46,47 \\
41 a 60 anos & 102 & 42,32 \\
61 anos ou mais & 27 & 11,20 \\
Estado civil & & \\
Com companheiro & 107 & 44,40 \\
$\quad$ Sem companheiro & 134 & 55,60 \\
Escolaridade & & \\
Ensino Fundamental & 69 & 28,63 \\
Ensino Médio & 75 & 31,12 \\
Ensino Superior & 97 & 40,25 \\
\hline
\end{tabular}

${ }^{*}$ Cinco consumidores tinham idade inferior a 18 anos, sendo excluídos do estudo.
Tabela 2. Associação entre características socioeconômicas e hábito de leitura dos rótulos. Pelotas-RS, 2011.

\begin{tabular}{|c|c|c|c|c|}
\hline \multirow{2}{*}{ Características } & \multicolumn{3}{|c|}{$\%$} & \multirow{2}{*}{$\mathbf{p}$} \\
\hline & Não & Sim & Às vezes & \\
\hline Gênero & & & & 0,052 \\
\hline Masculino & 40,74 & 42,59 & 16,67 & \\
\hline Feminino & 24,06 & 49,73 & 26,20 & \\
\hline Faixa etária & & & & 0,941 \\
\hline 18 a 40 anos & 28,57 & 48,21 & 23,21 & \\
\hline 41 a 60 anos & 25,49 & 49,02 & 25,49 & \\
\hline 61 anos ou mais & 33,33 & 44,44 & 22,22 & \\
\hline Estado civil & & & & 0,744 \\
\hline Com companheiro & 28,04 & 45,79 & 26,17 & \\
\hline Sem companheiro & 27,61 & 50,00 & 22,39 & \\
\hline Escolaridade & & & & 0,015 \\
\hline Ensino Fundamental & 30,43 & 43,48 & 26,09 & \\
\hline Ensino Médio & 37,33 & 36,00 & 26,67 & \\
\hline Ensino Superior & 18,56 & 60,82 & 20,62 & \\
\hline
\end{tabular}


mais criteriosas (MARINS et al., 2008; PINHEIRO et al., 2011).

Foi testada a associação entre hábito de leitura e influência das informações no ato da compra. Os resultados foram significativos, evidenciando que entre os consumidores que costumavam ler os rótulos, $62,07 \%$ ( $n=72$ ) relataram influência, enquanto que 19,83\% $(n=23)$ não sofriam influência e, em $18,10 \%(n=21)$, as informações influenciavam às vezes $(p=0,001)$. Estes achados mostram que as informações presentes são fundamentais no momento da aquisição dos alimentos; mostra-se, dessa forma, a importância da rotulagem em informar o consumidor sobre as propriedades nutricionais de um alimento.

Os rótulos são elementos identificadores e que, além da sua função publicitária, devem garantir ao consumidor um meio de informação que permita escolhas adequadas, auxiliando na decisão de compra e, consequentemente, aumentando a eficiência do mercado e o bem-estar do consumidor (MACHADO et al., 2006).

Em relação às informações mais procuradas, destacam-se: a data de validade (69,54\%); o valor calórico $(39,08 \%)$; o tipo e a quantidade de gordura $(30,46 \%)$; as vitaminas (16,67\%); o colesterol (14,94\%); se light, se diet (14,37\%); fibras (10,34\%); sódio (8,05\%); cálcio (5,75\%); alimentos específicos $(5,17 \%)$, e outras informações $(1,14 \%)$. A partir destes achados, pode-se concluir que o consumidor prioriza a informação relacionada à segurança do alimento, isto é, seu prazo de validade, uma vez que esta informação diz respeito ao tempo a partir do qual o alimento já não pode mais ser consumido sem danos à saúde do consumidor (MACHADO et al., 2006). Esse fato sugere que as campanhas de divulgação do Código de Defesa do Consumidor, que sedimentaram a obrigatoriedade da inclusão do prazo de validade nos rótulos, são eficazes (OLIVEIRA et al., 2005).

Em relação aos alimentos mais consultados (Tabela 3), o leite e seus derivados foram os que apresentaram as maiores prevalências, seguidos pelo grupo dos cereais e pelo grupo dos biscoitos.

Tabela 3. Principais alimentos que levam à consulta dos rótulos. Pelotas-RS, 2011

\begin{tabular}{lcc}
\multicolumn{1}{c}{ Alimentos } & $\mathbf{n}$ & $\%^{*}$ \\
Leite e derivados & 124 & 71,26 \\
Cereais/pães/grãos & 83 & 47,70 \\
Biscoitos/bolachas/petiscos & 83 & 47,70 \\
Embutidos/enlatados & 81 & 46,55 \\
Alimentos congelados & 70 & 40,23 \\
Refrigerantes & 25 & 14,37 \\
Chocolates & 22 & 12,64 \\
Sorvetes & 20 & 11,49 \\
\hline
\end{tabular}

*Os valores ultrapassam $100 \%$ porque os consumidores podem ter relatado mais de um alimento.
Dados semelhantes também foram encontrados por Monteiro et al. (2005), que apontaram que leite e derivados foram os alimentos mais consultados.

\section{Conclusão}

Pode-se concluir, pelo presente estudo, que a maioria dos consumidores possuía o hábito de ler os rótulos dos alimentos consumidos. Foi perceptível a leitura predominante entre o gênero feminino, em indivíduos jovens e com nível de escolaridade elevado. Além disso, a maioria dos entrevistados que consulta os rótulos, compreende as informações; no entanto, neste trabalho, isto não teve associação com o nível de escolaridade, evidenciando a necessidade de outros estudos para testar esta associação. Os dados também mostraram uma relação significativa entre o hábito de leitura e sua influência no momento da compra, inferindo o importante papel da rotulagem de alimentos na escolha dos alimentos.

Portanto, é de suma importância ressaltar a realização de outros estudos que avaliem o comportamento dos consumidores frente à rotulagem nutricional, com o intuito de identificar suas necessidades, com vistas à satisfação do cliente, priorizando, fundamentalmente, a saúde do consumidor.

\section{Referências}

AGÊNCIA NACIONAL DE VIGILÂNCIA SANITÁRIA - ANVISA; UNIVERSIDADE DE BRASÍLIA - UnB. Rotulagem Nutricional Obrigatória: Manual de Orientação às Indústrias de Alimentos. 2. versão. Brasília: ANVISA, UnB, 2005. 44 p.

BRASIL. Ministério da Justiça. Código de Defesa do Consumidor (CDC). Lei no 8.078/90, de 11 de setembro de 1990. Dispõe sobre a proteção do consumidor e dá outras providências. Diário Oficial da República Federativa do Brasil, Poder Executivo, Brasília, DF, 12 set. 1990. Seção 1. Disponível em: <http://www. planalto.gov.br/ccivil/LEIS/L8078.htm>. Acesso em ago. 2011.

BRASIL. Ministério da Saúde. Rotulagem geral de alimentos embalados. Resolução RDC n 359, de 23 de dezembro de 2003.

Diário Oficial da República Federativa do Brasil, Poder Executivo, Brasília, DF, 26 dez. 2003a. Seção 1. Disponível em: <http://www.anvisa.gov.br/alimentos/legis/especifica/rotuali. $\mathrm{htm}>$. Acesso em: ago. 2011.

BRASIL. Ministério da Saúde. Informação nutricional. Resolução RDC n $n^{\circ}$ 360, de 23 de dezembro de 2003. Diário Oficial da República Federativa do Brasil, Poder Executivo, Brasília, DF, 26 dez. 2003b. Seção 1. Disponível em: <http://www. anvisa.gov.br/alimentos/legis/especifica/rotuali.htm>. Acesso em: ago. 2011.

CÂMARA, M. C. C.; MARINHO, C. L. C.; GUILAM, M. C.; BRAGA, A. M. C. B. A produção acadêmica sobre a rotulagem de 
Rotulagem nutricional: você sabe o que está comendo?

CAVADA, G. S. et al.

alimentos no Brasil. Revista Panamericana de Salud Pública, Washington, v. 23, n. 1, p. 52-58, 2008. http://dx.doi.org/10.1590/ S1020-49892008000100007

CASSEMIRO, I. A.; COLAUTO, N. B.; LINDE, G. A. Rotulagem nutricional: quem lê e por quê? Arquivo Ciência Saúde UNIPAR, Umuarama, v. 10, n. 1, p. 9-16, 2006.

FELIPE, M. R.; MEZADRI, T.; CALIL, J. Rotulagem de alimentos: o comportamento do consumidor usuários de supermercados do balneário Camboriú/SC. Higiene Alimentar, São Paulo, v. 17, n. 111, p. 49-57, 2003.

GIMENO, S. G. A.; MONDINI, L.; MORAES, S. A.; FREITAS, I. C. M. Padrões de consumo de alimentos e fatores associados em adultos de Ribeirão Preto, São Paulo, Brasil: Projeto OBEDIARP. Caderno de Saúde Pública, Rio de Janeiro, v. 27 , n. 3, p. 533-545, 2011. http://dx.doi.org/10.1590/S0102311X2011000300013

LENZ, A.; OLINTO, M. T. A.; DIAS-DA-COSTA, J. S.; ALVES, A. L, BALBINOTTI, M.; PATTUSSI, M. P.; BASSANI, D. G. Socioeconomic, demographic and lifestyle factors associated with dietary patterns of women living in Southern Brazil. Caderno de Saúde Pública, Rio de Janeiro, v. 25, n. 6, p. 1297-306, 2009. http://dx.doi.org/10.1590/S0102-311X2009000600012

LOBANCO, C. M.; VEDOVATO, G. M.; CANO, C. B.; BASTOS, D. H. M. Fidedignidade de rótulos de alimentos comercializados no município de São Paulo, SP. Revista de Saúde Pública, São Paulo, v. 43, n. 3, p. 499-505, 2009. http://dx.doi.org/10.1590/ S0034-89102009005000020

MACHADO, S. S.; SANTOS, F. O.; ALBINATI, F. L.; SANTOS, L. P. R. Comportamento dos consumidores com relação à leitura de rótulo de produtos alimentícios. Alimentos e Nutrição, Araraquara, v. 17, n. 1, p. 97-103, 2006.

MARINS, B. R.; JACOB, S. C.; PERES, F. Avaliação qualitativa do hábito de leitura e entendimento: recepção das informações de produtos alimentícios. Ciência e Tecnologia de Alimentos, Campinas, v. 28, n. 3, p. 579-585, 2008. http://dx.doi. org/10.1590/S0101-20612008000300012

MONTEIRO, R. A.; COUTINHO, J. G.; RECINE, E. Consulta aos rótulos de alimentos e bebidas por frequentadores de supermercados em Brasília, Brasil. Revista Panamericana de Salud Pública, Washington, v. 18, n. 3, p. 172-177, 2005. http:// dx.doi.org/10.1590/S1020-49892005000800004

OLIVEIRA, M. J.; ARAÚJO, W. M. C.; BORGO, L. A. Parâmetros físico-químicos em linguiça do tipo frescal e avaliação das informações apresentadas no rótulo. Higiene alimentar, São Paulo, v. 19, n. 129, p. 47-56, 2005.

PINHEIRO, F. A.; CARDOSO, W. S.; CHAVES, K. F.; OLIVEIRA, A. S. B.; RIOS, S. A. Perfil de Consumidores em Relação à Qualidade de Alimentos e Hábitos de Compras. UNOPAR Cientifica. Ciências Biológicas e da Saúde, Londrina, v. 13, n. 2, p. 95-102, 2011

SILVA, M. Z. T. Influência da Rotulagem na Rotulagem Nutricional sobre o Consumidor. 2003. 67 f. Dissertação (Mestrado em Nutrição)-Universidade Federal de Pernambuco, Recife, 2003.

SOUZA, S. M. F. C.; LIMA, K. C.; MIRANDA, H. F.; CAVALCANTI, F. I. D. Utilização da informação nutricional de rótulos por consumidores de Natal, Brasil. Revista Panamericana de Salud Pública, Washington, v. 29, n. 5, p. 337-343, 2011. http://dx. doi. org/10.1590/S1020-49892011000500006 